\title{
Eficacia como constructo multidimensional en la determinación de estrategias de informatización empresarial
}

\author{
Effectiveness as a multidimensional construct in the determination \\ of strategies of enterprise informatization
}

\author{
Lisett Pérez Quintero ${ }^{1,2 *} \quad$ Jorge Carrera Ortega ${ }^{1,2} \quad$ Ana María García Pérez ${ }^{1,2}$ \\ Recibido 15 de diciembre de 2016, aceptado 31 de mayo de 2017 \\ Received: December 15, 2016 Accepted: May 31, 2017
}

\begin{abstract}
RESUMEN
La eficacia en la determinación de estrategias de informatización constituye un punto de análisis clave en la gestión organizacional. No solo es necesaria la evaluación de las posibles mejoras desde el punto de vista de la eficiencia y analizar la optimización de tiempo y recursos en las operaciones, también es importante lograr una integración sinérgica de los sistemas y los procesos concebidos a fin de tributar coherentemente a la misión de la organización. En el presente trabajo se describe un grupo de aspectos que tienen un carácter relevante dentro del dominio de contenido de la eficacia como variable. Se hace énfasis en el análisis de algunas dimensiones implicadas y sus interrelaciones. Para esto, se diseñó un instrumento de recopilación de información mediante la aplicación del método Delphi, para diagnosticar la relevancia de los factores identificados en un panel de 15 expertos. Se presenta, además, un indicador para medir la eficacia en la determinación de estrategias de informatización empresarial; basado en aspectos de la lógica difusa compensatoria (LDC) que considera la ocurrencia de factores de incertidumbre. La formulación de este indicador contribuye a la toma de decisiones sobre estrategias de informatización de manera que se produzcan reflexiones enfocadas a la mejora organizacional en su eficiencia y eficacia.
\end{abstract}

Palabras clave: Eficacia, estrategias de informatización.

\begin{abstract}
The effectiveness in the determination of informatization strategies constitutes a key analysis point in organizational management. It is not only necessary to evaluate possible improvements of efficiency and analyze the optimization of time and resources in operations, but it is also to achieve a synergistic integration of the systems and processes designed. In the present article, it is described a group of significant aspects concerning efficacy as a variable. Emphasis is given to the analysis of some implied dimensions and their interrelationships. An information collection instrument was designed using the Delphi method to diagnose the relevance of the factors identified in a panel of 15 experts. Based on aspects of compensatory fuzzy logic $(C F L)$ as an approach considering the occurrence of uncertainty factors, it is also presented a proposal for a general indicator for effectiveness in the determination of enterprise informatization strategies. The formulation of this indicator contributes to the decision making on informatization strategies in a way that produces reflections focused on the organizational improvement in its efficiency and effectiveness.
\end{abstract}

Keywords: Effectiveness, strategies of enterprise informatization.

1 Subdirección de Innovación y Desarrollo. DESOFT Villa Clara, Avenida Calixto García No 401 entre Carretera Central y Calle 4ta, Reparto Tirso Díaz, Santa Clara, Villa Clara, Cuba. E-mail: jorge.carrera@ vcl.desoft.cu; anamaria.garcia@ vcl.desoft.cu

2 Facultad de Matemática, Física y Computación. Universidad Central "Marta Abreu” de Las Villas (UCLV), Carretera a Camajuaní,

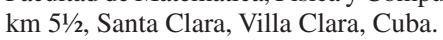

* Autor de correspondencia. E-mail: lisett.perez@vcl.desoft.cu 


\section{INTRODUCCIÓN}

Una estrategia de informatización empresarial constituye un conjunto de acciones claramente definidas en el tiempo, ordenadas por prioridad, medibles y alcanzables. Estas acciones consisten en la adquisición de aplicaciones informáticas con su adecuación correspondiente al entorno de uso, el desarrollo de aplicaciones a la medida, la compra de infraestructura de hardware y comunicaciones así como actividades de capacitación al personal. Para lograr obtener una correcta estrategia de informatización se debe utilizar un enfoque proactivo para la mejora de una organización en su eficiencia y eficacia. Su fundamentación es resultado de los análisis de factibilidad técnica, económica y operativa que se lleven a cabo sobre diversas alternativas atendiendo a los riesgos y oportunidades. Esta proyección aplica para el contexto empresarial a escala internacional, el que se caracteriza en la actualidad según la norma ISO 9000:2015 [1] por un cambio acelerado, los recursos limitados y la aparición del conocimiento como un recurso principal.

En la determinación de las estrategias de informatización se busca mejorar el desempeño organizacional mediante la optimización de los flujos de información, pero en la actualidad constituye un reto importante pensar en la mejora que se causa a los procesos organizacionales en su concepción integrada y sistémica. Hoy no es suficiente considerar criterios de optimización de tiempo y recursos desde funciones específicas y analizarlas por separado puede resultar caótico. Informatizar funciones de manera aislada propicia la aparición de problemas dentro de la concepción integrada que deben tener los sistemas de la calidad que, de hecho, abogan por el enfoque de procesos y no por los enfoques funcionales como modo de gestión.

El estudio realizado se centra en describir los factores fundamentales que deben tenerse en cuenta para la determinación de estrategias de informatización empresarial, considerando que el propósito de las mismas es la mejora de una organización en su eficiencia y eficacia. En este trabajo se asume como variable de análisis la eficacia.

Eficacia es un término ampliamente difundido en el contexto de las ciencias empresariales, que hace referencia al grado de cumplimiento del objetivo.
La eficiencia, por su parte, apunta a la manera en que se lleva a cabo el cumplimiento de los objetivos, indicando un análisis del uso de los recursos para su posible optimización.

Es importante poder medir esta eficacia que se aborda conceptualmente, y que se concibe relacionada al cumplimiento del objetivo de las estrategias de informatización. En el estudio de la metodología de la investigación científica se reconoce como constructo a "una variable medida y que tiene lugar dentro de una hipótesis, teoría o un esquema teórico. Es un atributo que no existe aislado sino en relación con otros" [2]. Al analizar la eficacia como constructo en la determinación de estrategias de informatización empresarial es posible reconocer que tiene dimensiones que se encuentran notoriamente vinculadas y que se conectan entre sí para formar lo que se reconoce como un constructo multidimensional (dimensiones que están centradas en la mejora de una organización en su eficiencia y eficacia). La validez de constructo puede verse afectada por una estrechez de contenido (que se excluyan dimensiones importantes), o amplitud exagerada (que contenga excesiva inclusión de otros constructos), por lo que se ha desarrollado en el presente trabajo una revisión de la literatura y una complementación con la aplicación del método Delphi para validar las dimensiones propuestas.

La concepción de este constructo puede contribuir a la toma de decisiones sobre estrategias de informatización de manera que se produzcan reflexiones más enfocadas a la mejora organizacional en su eficiencia y eficacia.

\section{ANTECEDENTES}

En el contexto de la gestión de la calidad, la norma ISO 9000:2015 [1] ha definido eficacia dentro de los términos relativos al resultado como el "grado en que se realizan las actividades planificadas y se logran los resultados planificados"; esta constituye un concepto que está esencialmente vinculado al desempeño empresarial y su mejora. Desempeño es otro término abordado en la ISO 9000:2015 [1], dentro de los términos relativos al resultado; es definido como "un resultado medible". Para concebir procesos de mejora en las organizaciones, por ejemplo al decidir estrategias de informatización, es determinante valorar el impacto en el desempeño. 
Dentro de la gestión empresarial un factor clave relacionado al desempeño es la gestión del capital humano. En el presente trabajo, coincidiendo con [3], se hace referencia a la gestión del capital humano considerando la persona como unidad biopsicosocial, que incluye gestionar el conocimiento. La gestión del capital humano y del conocimiento será referida en el presente trabajo como gestión del capital humano (GCH). Se toma por base a [3] ya que la GCH que en esa fuente se trata demanda consecuencia con el enfoque estratégico, sistémico, interdisciplinario, transdisciplinar, participativo, proactivo, de proceso y de competencias laborales. En este enfoque se encuentran elementos de valor tangible e intangible, considerando que "las personas y sus organizaciones son portadoras de valor intangible, significado por sus conocimientos, habilidades, motivaciones y valores".

Otro aspecto clave en el desempeño empresarial es el liderazgo y su percepción. El liderazgo se ha considerado como una competencia de gestión o gerencial [4] y se ha definido que constituye un modelo que otras personas desean seguir, que establece y mantiene relaciones con un amplio espectro de sujetos con el objetivo de entender las necesidades y conseguir apoyo, se anticipa a los conflictos y procura resolverlos por medio de soluciones adecuadas para todos, entre otras características. Se ha planteado que "las personas siguen a aquellas que son portadoras de satisfacción de sus necesidades e intereses" [5]. En la presente investigación, se ha considerado la importancia de lograr la adecuada atención a la percepción del liderazgo en la organización durante el análisis de propuestas de informatización. La correcta identificación del criterio de los líderes para analizar los problemas en los procesos y las alternativas de solución es un aspecto clave durante la determinación de estrategias de informatización. Este criterio ayuda en la identificación de las brechas en los procesos, permite determinar elementos motivacionales que influyen en el desempeño empresarial y que forman parte del grupo de aspectos a resolver dentro del marco organizacional.

La percepción de las perspectivas es también un aspecto fundamental de la gestión del capital humano que incide considerablemente en el desempeño empresarial. "A las perspectivas o esperanzas las consideramos de mucha importancia práctica" [3], en esa fuente se cita la apreciación del escritor inglés Bernard Shaw: "las personas no actuamos tanto por nuestras experiencias como por nuestras esperanzas". La manera en que la propuesta de informatización se adhiere a las perspectivas impacta en la usabilidad y aceptación de los participantes, los que son factores claves para la evaluación de los sistemas informáticos dentro de su aplicación práctica, según lo abordado en importantes estándares de calidad como la ISO/IEC 9126-1. Es claro que el conocimiento es esencial para que las personas puedan sentirse motivadas a trabajar en los cambios que implican las nuevas soluciones informáticas, siendo imprescindible aprender a utilizarlas correcta y sistemáticamente, pero si no se consideran adecuadamente las perspectivas existirá un factor de discrepancia motivacional que, de primera instancia, potenciaría un rechazo al cambio y la opinión desfavorable sobre la nueva concepción. Esto como consecuencia afectaría la usabilidad y dentro del proceso de capacitación requerido crearía problemas en el aprendizaje, instaurando un ciclo de rechazo en el que no se logra aprender y utilizar el nuevo conocimiento por problemas de motivación hacia el mismo, la falta de conocimiento a su vez potencia la desmotivación.

En [3] se expone que la percepción del liderazgo y la percepción de perspectivas son indicadores intangibles que tienen una correlación positiva y lineal con la productividad del trabajo y el aprovechamiento de la jornada laboral. Por lo que en los análisis de mejora deben considerarse ya que inciden en el desempeño organizacional; sin embargo, no son aspectos que se reconozcan formalmente en los análisis que con alta frecuencia se utilizan para informatizar procesos.

$\mathrm{Al}$ concebir mejoras mediante tecnologías informáticas generalmente se enfatiza en el impacto que tendrán las soluciones en la calidad de la información y los datos. Se analiza el flujo de la información considerando dónde se crea, actualiza, consulta o elimina. Pero existen otras condiciones que deben ser estimadas para una visión sistémica y estratégica de la organización que están relacionadas fundamentalmente a las funciones del responsable del proceso o la actividad. Existen diferencias respecto al significado de: dato e información. Según [6] la información "es el resultado de haber organizado o analizado los datos de alguna manera y con un 
propósito". Esa fuente también plantea que "cuanto más exacta es la información, tanto mayor su calidad y tanta mayor confianza pueden depositar los gerentes en ella para tomar decisiones". La eficacia del control en los procesos depende de la calidad de la información pero además de la posibilidad de tenerla en el tiempo oportuno. En [6] se hace referencia a la oportunidad de la información planteando que "la información ofrecida por el sistema de información debe estar al alcance de la persona indicada, en el momento oportuno", además aborda la relevancia de la información explicando que "la información que reciben los gerentes debe ser relevante para sus funciones y labores".

La norma NC ISO 9001: 2015 [7] emplea el enfoque a procesos, lo que incorpora el ciclo Planificar Hacer - Verificar - Actuar y el pensamiento basado en riesgos. El pensamiento basado en riesgos permite determinar los factores que podrían causar que los procesos no logren los resultados planificados. En el caso de una propuesta de informatización esto incluye el análisis de alternativas ante fallos del sistema automatizado, teniendo en cuenta los posibles efectos negativos, para determinar medidas preventivas al respecto y se hace necesario el establecimiento de controles.

En la NC ISO 9001:2015 [7] se plantea que la comprensión y gestión de los procesos interrelacionados como un sistema contribuye a la eficacia y la eficiencia de la organización en el logro de sus resultados previstos. Se expresa que el enfoque a procesos en un sistema de gestión de la calidad permite la mejora de los procesos con base en la evaluación de los datos y la información. Para el sistema de gestión de la calidad esa norma expresa que la organización debe determinar los procesos necesarios, las entradas requeridas y las salidas esperadas de estos procesos, determinar su secuencia e interacción; determinar y aplicar los criterios y los métodos (incluyendo el seguimiento, las mediciones y los indicadores del desempeño relacionados) necesarios para asegurarse de la operación eficaz y el control de estos procesos. Se deben determinar los recursos necesarios, asignar responsabilidades y autoridades para estos procesos; abordar los riesgos y oportunidades; evaluar estos procesos e implementar cualquier cambio necesario; entre otros aspectos [8]. Por tanto, para mantener el enfoque sistémico, los objetivos estratégicos de la informatización deben corresponderse con el enfoque a procesos y el sistema de gestión adoptado por la organización. Los enfoques actuales para la informatización; sin embargo, se centran en la factibilidad del cambio de acuerdo con perspectivas económicas, operacionales y técnicas de partes del negocio, donde muchos requisitos para el sistema de gestión quedan dispersos y no concertados sistémicamente como los riesgos, las responsabilidades, las interrelaciones entre procesos y las competencias.

\section{TRABAJOS RELACIONADOS}

Se realizó una revisión de la literatura, considerando como criterio de búsqueda estudios provenientes de cuatro núcleos de investigación: la administración de empresas, la planificación estratégica de las organizaciones, la alineación estratégica de las tecnologías de la información y el negocio, así como el enfoque de ingeniería de software orientado al valor.

El objetivo de esta revisión fue identificar criterios relacionados a la variable de estudio y distinguir una tendencia de contenido enfocando posibles dimensiones. Estos análisis posibilitan dar seguimiento a la validez de criterio y de contenido, que se abordan en [2] como requisitos en la recolección de datos mediante la aplicación de instrumento(s) de medición en el proceso investigativo.

Se observa en la literatura que se han considerado decenas de dimensiones para abordar la eficacia en la determinación de estrategias de informatización. Se hicieron estudios utilizando como apoyo el análisis formal de conceptos (FCA por sus siglas en inglés). Se decidió utilizar el FCA considerando sus potencialidades para las revisiones de la literatura donde se procede a analizar tendencias de contenidos en conceptos, como es el caso de [9].

En la Figura 1 se muestra como ejemplo un punto comparativo donde se seleccionaron doce referencias con diversidad de fecha y geografía, representados por: $[6,10,11,12,13,14,15,16,17,18,19,20]$.

La revisión permitió identificar lo siguiente:

- Existen destacadas publicaciones desde el marco de la ingeniería del software que abordan la eficacia en función del cumplimiento de 


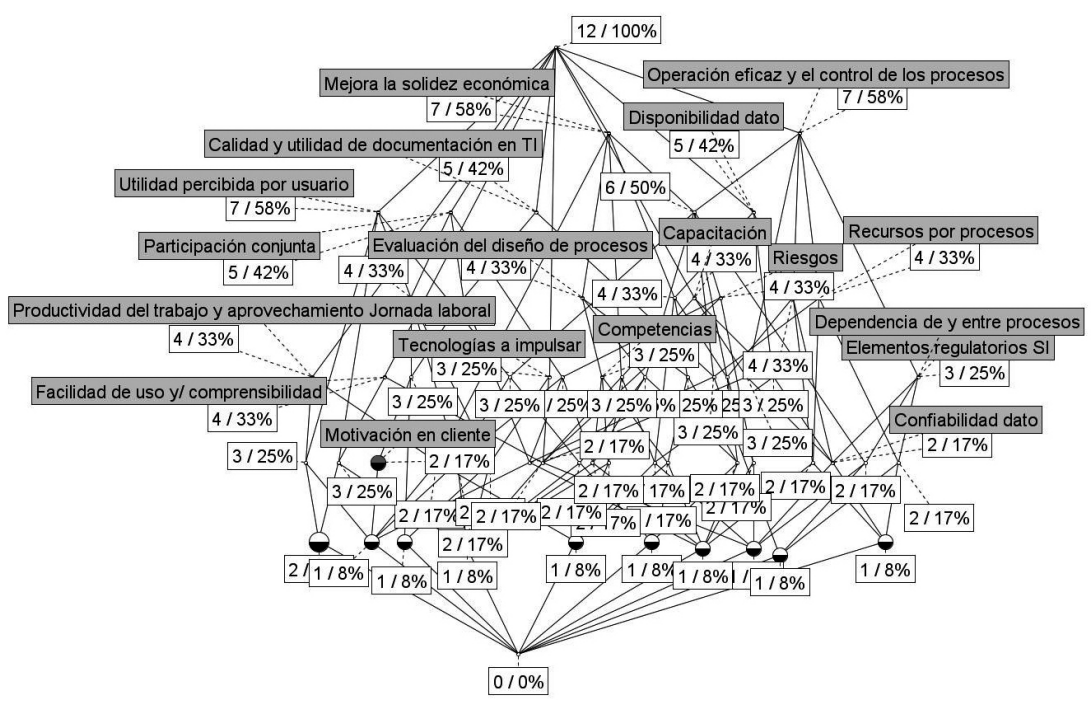

Figura 1. FCA de dimensiones de eficacia al decidir estrategias de informatización empresarial.

la satisfacción de los requisitos del cliente y sus expectativas, que no diagnostican si esos requisitos realmente están en consecuencia con la eficacia desde el punto de vista organizacional.

- No se refiere con significativo grado de generalización la existencia y aplicabilidad de un marco conceptual formalmente establecido para el constructo eficacia en la determinación de estrategias de informatización empresarial, que incluya un análisis dimensional al respecto.

- Existen dimensiones que resultan comunes, evidenciándose con mayor frecuencia: el análisis económico, la utilidad percibida por el usuario y el control de las operaciones.

\section{DETERMINACIÓN DE COMPONENTES Y DIMENSIONES DEL CONSTRUCTO EFICACIA}

Se decide aplicar un método Delphi para obtener una opinión de expertos consensuada y con consistencia mediante dos o más rondas sucesivas de ser necesarias, en relación al dominio de contenido de la variable eficacia en el contexto de la determinación de estrategias de informatización de empresas. Se opta por el método Delphi porque posibilita la consideración del criterio de experto de manera individual dentro del grupo, aislando la dificultad de la posible influencia de los criterios de un grupo de participantes en caso de realizar una exposición colectiva de la respuesta.
Primeramente se procedió a identificar qué características debían poseer los expertos. Para ello se realizó una encuesta a 12 investigadores que contaban con más de 15 años de labor en líneas de investigación relacionadas al análisis de mejora de procesos y su informatización. La encuesta pedía enunciar las características que debían poseer los participantes en un ejercicio de este tipo, según el objetivo de la encuesta. Como resultado las caracterización enunció: más de 10 años de experiencia de trabajo en dirección y gestión empresarial, resultados investigativos destacados en relación a la gestión empresarial (evidenciado en su participación en proyectos cerrados con éxito, participación como miembros de comité académico de eventos nacionales e internacionales y de maestrías relacionadas), experiencia en el análisis de informatización de procesos (publicaciones en revistas), que hayan asumido posiciones históricas relevantes relacionadas al estudio de la dirección empresarial (jefes de proyecto, decanos, directores de centros investigativos, jefes de departamentos docentes o de programas de maestrías). Para la selección de expertos se utilizó el análisis de síntesis curricular. Cumpliendo con esa caracterización se seleccionó un panel de 15 expertos y se desarrolló una ronda inicial con un cuestionario estructurado basado en preguntas abiertas sobre los componentes de la variable. A partir de los resultados de la primera ronda el grupo de análisis elaboró un segundo cuestionario con ítemes de escalamiento tipo Likert. 
Esta nueva encuesta se llevó a una segunda ronda con el propósito de obtener un criterio sobre la relevancia percibida en relación a cada componente y sus dimensiones para poder analizar el grado de consenso basado en los resultados de un análisis cuantitativo.

\section{Ronda primera}

En la primera ronda se utilizó un instrumento para la recolección de datos del tipo cuestionario; basado en preguntas abiertas. Se pidió mencionar cinco factores fundamentales por medio de los que se puede garantizar eficacia al decidir estrategias de informatización en las empresas (considerando eficacia en función de la misión de la empresa). Se realizó un proceso de análisis de los resultados para identificar los componentes enunciados y agrupar un conjunto de dimensiones. Este proceso requirió algunas entrevistas individuales para verificar la pertenencia del criterio arrojado por el investigador a los grupos definidos y erradicar repeticiones.

\section{Ronda segunda}

Para la segunda ronda se obtuvieron 11 componentes de la variable eficacia y un dimensionado para cada uno de ellos resultando en un total de 48 ítemes adicionales. Se elaboró un cuestionario para realizar una valoración de cada componente y dimensión utilizando un escalamiento tipo Likert. La escala diseñada es ordinal con cinco posibles posiciones de criterio. La evaluación se agrupó en los niveles de relevancia nula (0), muy baja - baja (1), regular (2), aceptable (3), elevada - total (4), indicando posiciones relativas en series ordenadas.

$\mathrm{Al}$ calcular el coeficiente de concordancia $C$ mediante $C=\left(1-\frac{v n}{V t}\right) \times 100$ donde $V n$ : cantidad de expertos en contra del criterio predominante y $V t$ : cantidad total de expertos, el resultado para cada componente indicó un buen nivel de consenso. Estos resultados se muestran en la Tabla 1. (Se considera buen nivel de consenso cuando es $>=60 \%$ acorde con [21]). $\mathrm{La}$ interpretación de las etiquetas $\mathrm{F}_{-} \mathrm{i}$ puede distinguirse con ayuda de la Figura 2.

En los estadísticos de los elementos se observa en la moda como medida de tendencia central que se obtiene un máximo valor: elevada - total (4) en los 11 ítemes (Figura 2). Sin embargo, en un grupo de ellos se reflejaba una variabilidad de criterio
Tabla 1. Coeficientes de concordancia.

\begin{tabular}{|c|c|c|c|}
\hline Factor & Moda & $V n$ & Coef. $C$ \\
\hline F_1 & 4 & 2 & 86,7 \\
\hline F_2 & 4 & 4 & 73,3 \\
\hline F_3 & 4 & 1 & 93,3 \\
\hline F_4 & 4 & 2 & 86,7 \\
\hline F_5 & 4 & 5 & 66,7 \\
\hline F_6 & 4 & 3 & 80,0 \\
\hline F_7 & 4 & 0 & 100,0 \\
\hline F_8 & 4 & 3 & 80,0 \\
\hline F_9 & 4 & 5 & 66,7 \\
\hline F_10 & 4 & 5 & 66,7 \\
\hline F_11 & 4 & 4 & 73,3 \\
\hline
\end{tabular}

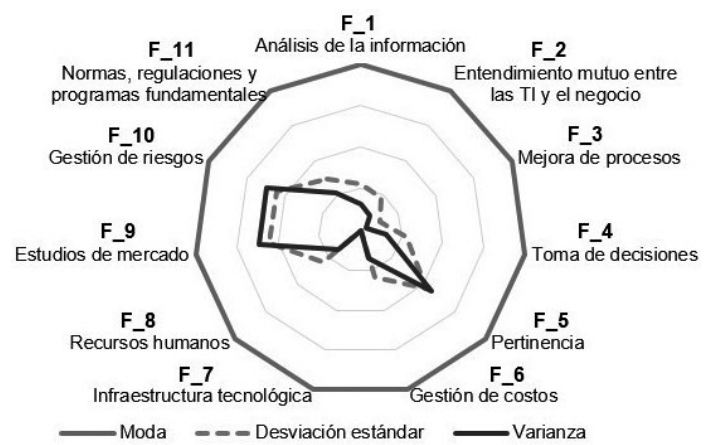

Figura 2. Componentes de eficacia al decidir estrategias de informatización.

importante a considerar, teniendo en cuenta las dimensiones expresadas para cada componente.

En los componentes mejora de procesos, e infraestructura tecnológica localiza el menor índice de desviación estándar al revisar las medidas de dispersión en los criterios emitidos. En el caso de infraestructura tecnológica con una desviación estándar y una varianza de 0,00 , y la mejora de procesos con una varianza de 0,07 .

Los componentes: gestión de riesgos, estudios de mercado y pertinencia obtuvieron los valores de desviación estándar y varianza más elevados indicando cuán alejado están los valores de la distribución del valor representativo resultante.

Se diagnosticó, por medio de un análisis llevado a cabo con el panel de expertos, que la variabilidad del criterio evidenciada tiene relación con la 
interpretación de cada componente desde el punto de vista de las dimensiones que puede contener.

Análisis de pertinencia, gestión de riesgos, estudios de mercado en relación dimensional con la mejora de procesos

Pertinencia, gestión de riesgos y estudios de mercado surgen como componentes en la primera ronda pero son los que obtuvieron mayor variabilidad en la segunda ronda.

Pertinencia constituyó una variable asociada al concepto de propósito. Este aspecto indica que algo es oportuno, en consecuencia a la intención y al ánimo. Es entonces que, por alegar una extensión motivacional, se mantuvo como un componente para estudiar sus posibles inclusiones. La dimensión que expresaba pertinencia en relación a la eficacia del proceso resultó ser la de mayor valor atribuido para su consideración (media), $C=86,7 \%$. Para este caso los valores de variabilidad siendo los menores entre todas las dimensiones dadas en ese componente fueron aun así muy altos (desviación estándar de 1,047 y varianza de 1,095); el grupo de análisis constató que se expresaba con ese ítem la relación conceptual de pertinencia y eficacia, pero no destacaba como un componente del constructo.

En el componente gestión de riesgos, la dimensión relacionada a la reducción de riesgos en los procesos con la propuesta de informatización fue la de mayor valor otorgado para su consideración (media) $C=86,7 \%$. Para este caso los valores de variabilidad siendo los menores entre todas las dimensiones dadas en el componente, fueron sin embargo altos (desviación estándar de 0,799 y varianza de 0,638 ). De la misma manera, los elementos reflejados en estudios del mercado resultaron con una alta variabilidad. La dimensión relacionada a la flexibilidad y capacidad de reacción exigida por el mercado a sus productos y servicios resultó tener los valores de variabilidad más bajos en relación a las demás dimensiones analizadas en este componente, siendo también la de mayor valor atribuido para su consideración (media), $C$ $=66,7 \%$; sin embargo, los valores de variabilidad fueron altos.

$\mathrm{Al}$ analizar la fluctuación de los criterios se pudo concluir que gestión de riesgos y estudios de mercado son de alta importancia, pero no como componentes separados sino que se debían considerar comprendidos en las dimensiones del componente mejora de procesos. Específicamente porque la reducción de riesgos en los procesos con la propuesta de informatización, así como flexibilidad y capacidad de reacción exigida por el mercado a sus productos y servicios son elementos claves para que la propuesta de informatización esté integrada al sistema de gestión de la calidad en la organización (aspecto este que se consideró como dimensión de mejora de procesos con alto consenso, $C=93,3 \%$. Para el caso del componente mejora de procesos se puede observar un alto consenso además en las dimensiones: la información más los recursos TI añadan valor al negocio en el cumplimiento de su misión, $C=100 \%$; disminución de costos o incremento de las ganancias que pueda lograr la empresa con la informatización de sus procesos, $C=73,3 \%$; mejora del sistema de control, $C=73,3 \%$; la inversión debe hacerse en el recurso limitante (el que impide mayor eficacia), $C=73,3 \%$.

La participación conjunta como dimensión relevante en el entendimiento mutuo entre las TIC y el negocio

El componente entendimiento mutuo entre las TIC y el negocio se consideró como un factor de alta importancia a tener en cuenta dentro de la variable eficacia para la determinación de estrategias de informatización. Este componente hace referencia a la manera en la que se logra una comunicación adecuada entre los analistas informáticos y los especialistas de negocio para la identificación del problema a resolver, las expectativas y la determinación de alternativas de solución.

Este componente obtuvo en la segunda ronda altos valores en los resultados de análisis de la media, mediana y moda. La concordancia sobre la inclusión del ítem como componente fue aceptable. Sin embargo, se observaba dispersión en relación a la descripción de varias dimensiones. Se pudo observar un consenso alto sobre la dimensión participación conjunta entre los especialistas del negocio y los analistas informáticos. Los ítemes entrenamiento y aprendizaje compartido obtuvieron, por criterios de tendencia central una evaluación alta; pero con alta varianza. Al analizar las causas de la alta varianza, se determinó que ambos aspectos deben considerarse como parte del componente recursos humanos. 
El conocimiento y las competencias en el componente recursos humanos

Se identificó la dimensión: se tengan en cuenta los conocimientos y habilidades del personal que manipulará los sistemas a fin de proyectar la capacitación que se les debe brindar, $C=86,7 \%$. Dentro de este componente se ubicó además el aspecto de percepción del liderazgo y el de la percepción de las perspectivas, en concordancia con lo tratado sobre la contribución al aumento del desempeño empresarial que se logra a partir de la gestión del capital humano, ya que esto está relacionado a la gestión de competencias (liderazgo y motivación), considerar las competencias del personal tuvo $C=80 \%$.

Este componente, revisado como recursos humanos, se abordará como "contribución a la productividad del trabajo y al aprovechamiento de la jornada laboral en la gestión del capital humano”.

Gestión de costo y la consideración de la infraestructura tecnológica

Dentro del componente gestión de costo se analizaron varias dimensiones y se logró consenso en relación costo-beneficio alcanzable con la informatización, $C=66,7 \%$ y en mayor consenso el aspecto: hacer el proyecto y evaluar alternativas de inversión para tomar decisiones, $C=80 \%$.

En el componente infraestructura tecnológica, como se había mencionado anteriormente, localiza el menor índice de desviación estándar al revisar los criterios emitidos (desviación estándar y una varianza de 0,00). Las dimensiones propuestas constan de la consideración de la infraestructura tecnológica existente para que se asimile adecuadamente la propuesta de informatización desde el punto de vista operacional y técnico, $C=93,3 \%$ y la integración al resto de los sistemas de gestión, $C=80 \%$ lo que se consideró parte de la dimensión integración al sistema de gestión organizacional.

De acuerdo con lo anterior, se definió para mejora de procesos una dimensión llamada mejora de la solidez económica, donde se incluye hacer el proyecto y evaluar alternativas de inversión para tomar decisiones; en esto queda contemplada la necesidad de consideración de la infraestructura tecnológica existente para el análisis de factibilidad. Se definió además el ítem existe disminución de costos (lo que implica mejoras del estado financiero) o incremento de la rentabilidad/ganancias que pueda lograr la empresa con la informatización de sus procesos (aumento de las ventas como parte de mejora de la solidez económica).

Análisis de la información, la toma de decisiones y la consideración de las normas, regulaciones, así como los programas fundamentales

En el componente propuesto como análisis de la información se logró una alta concordancia en las dimensiones confiabilidad de los datos (manejo de información), $C=93,3 \%$ y disponibilidad de los datos (manejo de información), $C=86,7 \%$ con una muy baja varianza de 0,07 y 0,12 respectivamente. $\mathrm{El}$ aspecto garantizar que cada dato primario se capture una sola vez obtuvo una alta consideración, $C=73,3 \%$ asumiéndose como fundamento para la confiabilidad de los datos.

Al analizar los ítemes dentro del componente: toma de decisiones, se evidenció alta variabilidad, y un excelente consenso $(C=93,3 \%)$ resultó en la dimensión: Calidad de la información en función de la toma de decisiones (varianza de 0,07). Considerando este resultado, el grupo de análisis decidió no contemplar toma de decisiones como un componente aparte sino dentro del componente análisis de la información mediante las dimensiones confiabilidad y disponibilidad de los datos (ver Figura 3).

Análisis de la información fue considerada como una dimensión compuesta del componente mejora de procesos; teniendo en cuenta que esta ya estaba contemplada en este componente como "la información más los recursos de TI utilizados añadan valor al negocio en el cumplimiento de su misión”, dimensión resultante en $C=100 \%$.

En normas, regulaciones y programas fundamentales se logra una concordancia muy positiva. En el análisis para identificar sus dimensiones se alcanza una alta concordancia de criterio respecto a la correspondencia con el programa rector de la informatización de la sociedad $C=86,7 \%$. También que se consideran los criterios necesarios (ej., normas y regulaciones vigentes) para asegurarse de la operación eficaz y el control de los procesos a informatizar fue un aspecto con alta concordancia $C=86,7 \%$. Ambas 


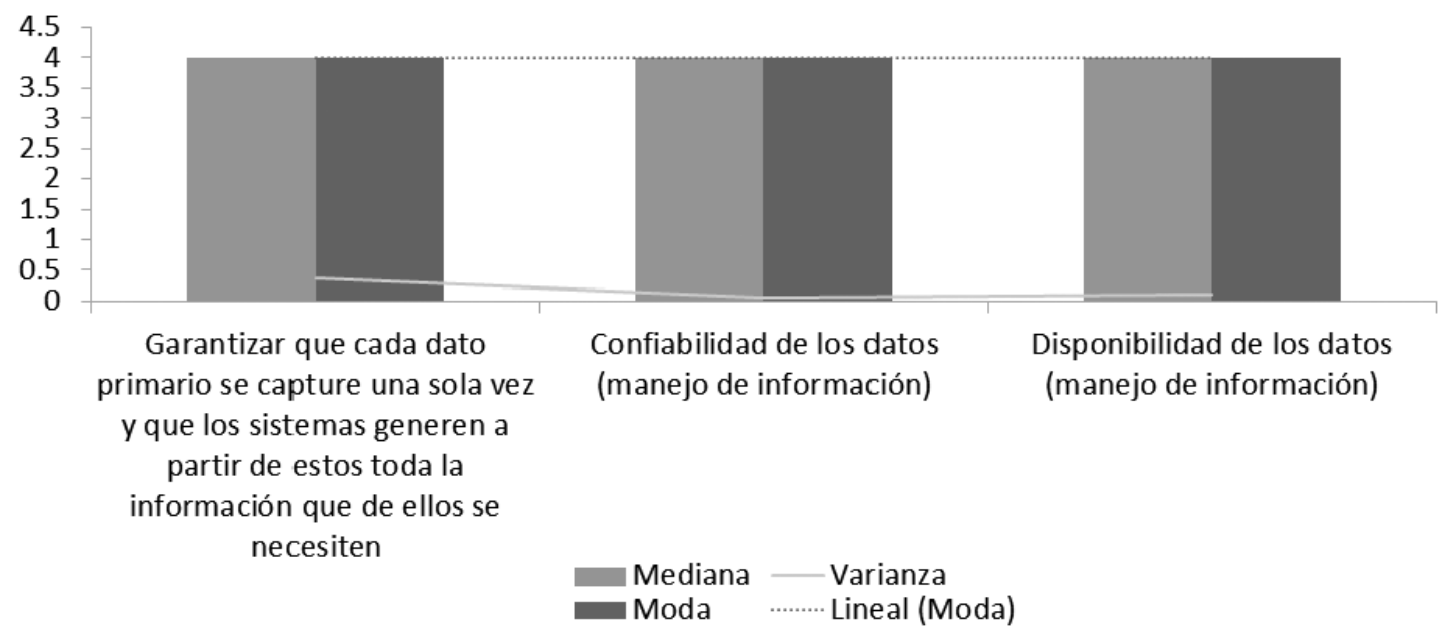

Figura 3. Dimensiones destacadas del componente análisis de la información.

propuestas de dimensiones eran propensas a confluir en este mismo componente, y se decidió con el grupo de expertos incluir en mejora de procesos la dimensión referente a que se consideran los criterios necesarios para asegurarse de la operación eficaz y el control de los procesos.

\section{Evaluación de la confiabilidad del instrumento} Para evaluar la confiabilidad del instrumento se opta por una medida de consistencia interna como es el caso del coeficiente alfa de Cronbach. Una vez aplicado el instrumento a la muestra se calculó el coeficiente obteniendo los siguientes resultados.

\section{a) Coeficiente alfa de Cronbach para los 11 componentes.}

Se evaluaron los once componentes de la variable. En el análisis de fiabilidad se optaron por estadísticos determinados, como el caso descriptivo para el elemento, la escala y una valoración de la escala si se elimina el elemento. También se consideraron los interelementos: correlación y covarianza, arribando a un resumen de las medias, varianza, covarianza y correlaciones.

El valor de alfa indica el grado de confiabilidad del instrumento, considerando una escala de 0 a 1 , a medida que se acerca a 1 es sumamente confiable la medida de la variable, siendo 1 el mayor valor teórico de alfa. En este caso el alfa de Cronbach resultó 0,823 , para los once componentes. Esto evidencia una alta fiabilidad del índice.

\section{b) Coeficiente alfa de Cronbach para las 48} dimensiones.

Se evaluaron las 48 dimensiones junto a los once componentes de la variable, siendo estos los ítemes generales declarados al dominio de contenido de eficacia. En este caso el alfa de Cronbach resultó 0,951, para los 59 elementos (11 componentes y 48 dimensiones). Esto evidencia una alta fiabilidad del índice para la medición general.

El coeficiente de homogeneidad corregido (correlación elemento-total corregida) para ningún ítem tuvo un valor de cero o negativo. El cálculo del alfa de Cronbach se realizó también probando la eliminación de los ítemes de manera individual, con la eliminación de ningún ítem específicamente se obtiene un alfa de Cronbach significativamente superior al obtenido con todos los componentes considerados en el instrumento.

\section{Indicador de la eficacia al decidir estrategias de informatización}

El constructo estudiado tiene un carácter multidimensional y algunas de sus dimensiones se componen a su vez de otros aspectos. Además, los valores de los aspectos tienen carácter difuso puesto que en cada contexto tienen presencia de incertidumbre. Por esta razón se decide la utilización de la Lógica Difusa Compensatoria (LDC) [22], en la formulación de un indicador para medir la variable estudiada. Se consideraron varios predicados difusos $p$ (expresiones lingüísticas) con un grado de verdad 
dentro de un intervalo [0,1], considerando que estas proposiciones responden a cierta condición de incertidumbre. Se formulan determinados predicados difusos simples, enunciados como $p s$ y los grados de verdad como $\mu_{p s}$ para cada expresión declarada. Los predicados compuestos son referidos por $p c$, representando la combinación de varios $p s \mathrm{u}$ otros $p c$ y los grados de verdad como $\mu_{p c}$.

Teniendo el resultado del ejercicio con expertos, anteriormente detallado, se arriba a lo siguiente:

\section{Enunciado verbal:}

"Se logra alta eficacia en la determinación de estrategias de informatización si la mejora de procesos considerada es alta, la participación conjunta entre los especialista de TI y del negocio es alta, se concibe una contribución a la productividad del trabajo y al aprovechamiento de la jornada laboral en la gestión del capital humano y el grado de correspondencia con el programa rector de la informatización de la sociedad es alto".

1. La mejora de procesos considerada es alta si la información más los recursos de TI utilizados añaden valor al negocio en el cumplimiento de su misión mejorando el sistema de control; la propuesta de informatización se analiza enfocada desde el sistema de gestión de la calidad en la organización; y se mejora la solidez económica.

1.1. La información más los recursos de TI utilizados añaden valor al negocio en el cumplimiento de su misión mejorando el sistema de control si se analiza el flujo de información procurando mejorar en el hecho de que cada dato primario se capture una sola vez tributando a la confiabilidad de los datos; y se busca mejora en la disponibilidad de los datos considerando que la inversión debe hacerse en el recurso limitante (el que impide mayor eficacia).

1.1.1. Se mejora la disponibilidad de los datos considerando que la inversión debe hacerse en el recurso limitante (el que impide mayor eficacia) si existe mejor oportunidad de información y además mejor relevancia en la información obtenida de acuerdo a las funciones del responsable del proceso/actividad.

1.2. La propuesta de informatización se analiza enfocada desde el sistema de gestión de la calidad en la organización si se repasan las relaciones de dependencia de y entre los procesos; se consideran los criterios y métodos necesarios para asegurarse de la operación eficaz y el control de los procesos; se consideran los recursos por procesos; se evidencia el pensamiento basado en riesgos en el análisis de la propuesta de informatización; se considera la evaluación del diseño de los procesos y se apoya el análisis para la implementación de cambios necesarios para que los procesos logren los resultados previstos (considerando el estudio de alternativas).

1.3 Se mejora la solidez económica si existe disminución de costos (lo que implica mejoras del estado financiero) o incremento de la rentabilidad/ganancias que pueda lograr la empresa con la informatización de sus procesos (aumento de las ventas) y para este análisis se haga el proyecto y se evalúen alternativas de inversión para tomar decisiones.

2. La participación conjunta entre los especialistas de TI y los especialistas de negocio en el análisis de la mejora es alta si se logra trabajar con herramientas para el análisis que resulten de fácil comprensibilidad por todos los involucrados, se motive el intercambio de criterios en la identificación del problema y en las propuestas de solución.

3. Se concibe una contribución a la productividad del trabajo y al aprovechamiento de la jornada laboral en la gestión del capital humano si existe una adecuada influencia en la percepción de perspectivas respecto a la estrategia de informatización; se realice una atención a la percepción del liderazgo en la organización; se tengan en cuenta los conocimientos y habilidades del personal que manipulará los sistemas a fin de proyectar la capacitación que se les debe brindar.

4. El grado de correspondencia con el programa rector de la informatización de la sociedad es alto si se cumple con los elementos regulatorios en relación a los aspectos de seguridad informática comprendidos; se cumple con los principios en relación con los aspectos éticos en el uso de las tecnologías; se tienen en cuenta las prioridades de tecnologías informáticas a impulsar.

\section{Predicados:}

Se considera que se logra una determinación eficaz de una estrategia de informatización si:

$p c=$ "La mejora de procesos considerada es alta" (1). 


$$
\mu_{p c}=M P C
$$

$p c=$ "La participación conjunta entre las TI y el negocio es alta" (2).

$$
\mu_{p c}=P C T N
$$

$p c=$ "Se concibe una contribución a la productividad del trabajo y al aprovechamiento de la jornada laboral en la gestión del capital humano" (3).

$$
\mu_{p c}=C G C H
$$

$p c=$ "El grado de correspondencia con el programa rector de la informatización de la sociedad es alto" (4).

$$
\mu_{p c}=C P R I S
$$

El predicado compuesto del modelo general es el siguiente $\left(\mu_{p c}=E D E I\right)$, valor de verdad del nivel de eficacia en la determinación de una estrategia de informatización para empresas (5).

$$
E D E I=M P C \cap P C T N \cap C G C H \cap C P R I S
$$

Las expresiones lingüísticas de cada predicado compuesto fueron analizadas y detalladas arribando a una representación de estructura de árbol, utilizando conectores lógicos para la asociación de los nodos. El nivel de desglose permitió partir de una raíz en el árbol que representaba el predicado compuesto general y arribar a niveles de predicados compuestos hasta obtener hojas con predicados simples según los enfoques de LDC expuestos en [22], fuente que además aborda las posibilidades de la herramienta "Fuzzy Tree Studio" para enfoques de este tipo.

Se considera para $p c=$ "La mejora de procesos considerada es alta” $\left(\mu_{p c}=M P C\right)$ si (6):

$$
M P C=I T I V C \cap I S G O \cap M S E
$$

- Descripción de ITIVC dentro de (6)

$p c=$ "La información más los recursos de TI utilizados añaden valor al negocio en el cumplimiento de su misión mejorando el sistema de control" (7), detallado en (8).

$$
\mu_{p c}=I T I V C
$$

$$
I T I V C=C D U \cap D D R L
$$

Para (8) se considera (9) y (10).

$p s=$ "Se analiza el flujo de información procurando mejorar en el hecho de que cada dato primario se capture una sola vez tributando a la confiabilidad de los datos"(9).

$$
\mu_{p s}=C D U
$$

$p c=$ "Se busca mejora en la disponibilidad de los datos considerando que la inversión debe hacerse en el recurso limitante"(10), detallado en (11).

$$
\mu_{p c}=D D R L
$$

$$
D D R L=O P I \cap R I O
$$

Para (11) se considera (12) y (13).

$p s=$ "Existe mejor oportunidad de información"(12)

$$
\mu_{p s}=O P I
$$

$p s=$ "Mejor relevancia en la información obtenida de acuerdo a las funciones del responsable del proceso/actividad" (13)

$$
\mu_{p s}=R I O
$$

Descripción de $I S G O$ dentro de (6)

$p c=$ "La propuesta de informatización se analice enfocado desde el sistema de gestión de la calidad en la organización"(14), detallado en (15)

$$
\mu_{p c}=I S G O
$$

$$
I S G O=R D P \cap O E C P \cap R P \cap P B R \cap A I C
$$

Para (15) se considera (16), (20), (25), (29) y (33): $p c=$ "Se repasen las relaciones de dependencia de $y$ entre los procesos"(16), detallado en (17). 


$$
\begin{gathered}
\mu_{p c}=R D P \\
R D P=E R S E \cap S I P
\end{gathered}
$$

Para (17) se considera (18) y (19):

$p s=$ "Se identifiquen las entradas requeridas y salidas esperadas de los procesos”(18)

$$
\mu_{p s}=E R S E
$$

$p s=$ "Se determine la secuencia e interacción de los procesos"(19)

$$
\mu_{p s}=S I P
$$

$p c=$ "Se consideren los criterios y métodos necesarios para asegurarse de la operación eficaz y el control de los procesos"(20), detallado en (21)

$$
\begin{gathered}
\mu_{p c}=O E C P \\
O E C P=I D R \cap D R P \cap A E P
\end{gathered}
$$

Para (21) se considera (22), (23) y (24):

$p s=$ "Se consideran las variables de control relacionados al proceso con los indicadores de desempeño"(22).

$$
\mu_{p s}=I D R
$$

$p s=$ "Se consideran los documentos que rigen como mecanismos de control a los procesos" (23).

$$
\mu_{p s}=D R P
$$

$p s=$ "Se consideran las actividades de evaluación como parte de la descripción del proceso"(24).

$$
\mu_{p s}=A E P
$$

$p c=$ "Se considere los recursos por procesos" (25), detallado en (26).

$$
\begin{gathered}
\mu_{p c}=R P \\
R P=R N P D \cap R E A U
\end{gathered}
$$

Para (26) se considera (27) y (28):

$p s=$ "Se considere los recursos necesarios para los procesos y su disponibilidad” (27).

$$
\mu_{p s}=R N P D
$$

$p s=$ "Se considere las responsabilidades y autoridades para estos procesos”(28).

$$
\mu_{p s}=R E A U
$$

$p c=$ "Se evidencie el pensamiento basado en riesgos en el análisis de la propuesta de informatización" (29), detallado en (30).

$$
\begin{gathered}
\mu_{p c}=P B R \\
P B R=P E N D \cap P A R
\end{gathered}
$$

Para (30) se considera (31) y (32):

$p s=$ "Se prevean los efectos no deseados de la propuesta de informatización” (31).

$$
\mu_{p s}=P E N D
$$

$p s=$ "Se planifiquen, en el proceso, las acciones para abordar los riesgos de la propuesta de informatización” (32).

$$
\mu_{p s}=P A R
$$

$p c=$ "Se considere la evaluación del diseño de los procesos y se apoya el análisis para la implementación de cambios necesarios para que los procesos logren los resultados previstos (Considerando el estudio de alternativas)" (33) detallado en (34).

$$
\mu_{p c}=A I C
$$

$$
A I C=S P A S \cup S P A C B \cup S P A P M
$$

Para (34) se considera (35), (36) y (37):

$p s=$ "Se subsanen procesos con actividades superfluas” (35).

$$
\mu_{p s}=S P A S
$$


$p s=$ "Se subsanen procesos con cuellos de botella" (36).

$$
\mu_{p s}=S P A C B
$$

$p s=$ "Se subsanen procesos con deadlock (puntos muertos)” (37).

$$
\mu_{p s}=S P A P M
$$

Descripción de MSE dentro de (6)

$p c=$ "Se mejora la solidez económica" (38), detallado en (39).

$$
\begin{gathered}
\mu_{p c}=M S E \\
M S E=D C O \cup I N R
\end{gathered}
$$

Para (39) se considera (40) y (41):

ps = "Existe disminución de los costos" (40).

$$
\mu_{p s}=D C O
$$

ps = "Incremento de la rentabilidad" (41).

$$
\mu_{p s}=I N R
$$

Se considera para $p c=$ "La participación conjunta entre las TI y el negocio es alta” ( $\mu_{p c}=$ PCTN) si (42):

$$
P C T N=H F C \cap M I N C P \cap M I N C S
$$

Para (42) se considera (43), (44) y (45).

$p s=$ "Se logra trabajar con herramientas para el análisis que resulten de fácil comprensibilidad por todos los involucrados" (43).

$$
\mu_{p s}=H F C
$$

$p s=$ "Se motive el intercambio de criterios en la identificación del problema" (44).

$$
\mu_{p s}=\operatorname{MINCP}
$$

$p s=$ "Se motive el intercambio de criterios en la identificación de las propuestas de solución” (45).

$$
\mu_{p s}=\operatorname{MINCS}
$$

Se considera para $p c=$ "Se concibe una contribución a la productividad del trabajo y al aprovechamiento de la jornada laboral en la gestión del capital humano" $\left(\mu_{p c}=C G C H\right)$ si (46):

$$
C G C H=P P E I \cap A P L O \cap C H P C
$$

Para (46) se considera (47), (48) y (49).

$p s=$ "Existe una adecuada influencia en la percepción de perspectivas respecto a la estrategia de informatización" (47).

$$
\mu_{p s}=P P E I
$$

$p s=$ "Se realice una atención a la percepción del liderazgo en la organización” (48).

$$
\mu_{p s}=A P L O
$$

ps = "Se tengan en cuenta los conocimientos y habilidades del personal que manipulará los sistemas a fin de proyectar la capacitación que se les debe brindar" (49).

$$
\mu_{p s}=C H P C
$$

Se considera para $p c=$ "El grado de correspondencia con el programa rector de la informatización de la sociedad es alto si” $\left(\mu_{p s}=\right.$ CPRIS) si (50):

$$
C P R I S=E R S I \cap A E U T \cap P T I
$$

Para (50) se considera (51), (52) y (53).

$p s=$ "Se cumple con los elementos regulatorios en relación a los aspectos de seguridad informática comprendidos" (51).

$$
\mu_{p s}=E R S I
$$

$p s=$ "Se cumple con los principios en relación a los aspectos éticos en el uso de las tecnologías" (52).

$$
\mu_{p s}=A E U T
$$

$p s=$ "Se tienen en cuenta las prioridades de tecnologías informáticas a impulsar" (53). 


$$
\mu_{p s}=P T I
$$

Se propone trabajar con la siguiente escala:

1) 0: completamente falso

2) 0,25: más falso que verdadero;

3) $0,5:$ ni falso ni verdadero;

4) 0,75: más verdadero que falso;

5) 1: completamente verdadero

\section{CONCLUSIONES Y TRABAJO FUTURO}

En el presente trabajo se identificaron componentes de la eficacia como constructo multidimensional en la determinación de estrategias de informatización empresarial. En el proceso se realizó un ejercicio con expertos de la rama de la gestión empresarial lográndose arribar a un consenso y se realizó un análisis formal de conceptos en la revisión de la literatura para reflexionar sobre la validez de criterio y de contenido lograda en las rondas Delphi con los expertos.

Los resultados demostraron variabilidad respecto al concepto eficacia en la determinación de estrategias de informatización empresarial, diagnosticado en la revisión de la literatura mediante el FCA y además como resultado de la primera ronda ejercida para el método Delphi, donde los criterios abiertos fueron tan diversos que llevaron a la propuesta inicial de 11 componentes y 48 dimensiones.

Se pudo constatar que si no se considera adecuadamente el carácter multidimensional de este concepto se establecerían muchos y diferentes parámetros como determinantes, soslayando el enfoque sistémico en la organización y el valor de un adecuado enfoque a procesos.

Las dimensiones propuestas difieren respecto a las identificadas en la literatura por su concepción combinada para abordar la determinación de estrategias de informatización empresarial considerando que el objetivo es la mejora de una organización en su eficiencia y eficacia. Es posible encontrar en la literatura la relevancia de cada una de las dimensiones propuestas; sin embargo, se enuncian dispersas y no como parte del mismo constructo. Una mayor coincidencia se detectó en la mejora de la solidez económica, operación eficaz y control de los procesos, así como la participación conjunta de los especialistas de TI y los especialistas del negocio, pero aun así el tratamiento a cada uno de estos elementos tiene en la literatura diferentes enfoques.

El coeficiente alfa de Cronbach evidenció una alta fiabilidad del instrumento utilizado en la aplicación del método Delphi. Lo que evidencia que es posible llegar a un consenso aun cuando existe como punto de partida una gran diversidad de criterios. El carácter multidimensional del concepto propició establecer puntos de organización de las ideas y declarar un contenido no excluyente para los factores concebidos como claves.

El resultado fue enunciado verbalmente y se propuso un indicador basado en lógica difusa compensatoria para poder llevar a cabo análisis de estos componentes en el marco organizacional, como apoyo en la evaluación de estrategias de informatización empresarial.

Como trabajo futuro de investigación se encuentra la consideración de una propuesta para el modelado organizacional que aborde las dimensiones de los componentes identificados, en busca de la facilitación de reflexiones necesarias desde la base del entendimiento mutuo de los especialistas de TI y del negocio; además, la elaboración de una herramienta informática que soporte esa propuesta de modelado. Esto propiciaría una continuidad de la investigación abordando la eficiencia, la que estaría centrada en el modo de cumplimiento de los objetivos que están ya definidos en las dimensiones de eficacia que se han identificado.

\section{REFERENCIAS}

[1] Oficina Nacional de Normalización (NC). "Sistemas de gestión de la calidadFundamentos y vocabulario. [ISO 9000: 2015 (Traducción certificada), IDT]", Ciudad de La Habana, Oficina Nacional de Normalización (NC). 2015.

[2] R. Hernández Sampieri, C. Fernández Collado y M.d.P. Baptista Lucio. "Metodología de la investigación". Quinta edición, México: McGrawHill. 2010.

[3] A. Cuesta Santos y M. Valencia Rodríguez. "Indicadores de Gestión del Capital Humano y del Conocimiento en la empresa". La Habana: Academia. 2014. 
[4] R. Lorenzo García. "Talento, éxito y liderazgo", La Habana: Editorial CientíficoTécnica. 2008.

[5] G.A. Ronda Pupo. "Dirección estratégica, Constructo y Dimensiones". Ciudad de La Habana, Cuba: Ediciones Futuro, 2007.

[6] J.A.F. Stoner, E. Freeman y D.R. Gilbert. "Administración", México: Prentice Hall, 1996.

[7] Oficina Nacional de Normalización (NC). "Sistemas de Gestión de la Calidad Requisitos [ISO 9001:2015 (Traducción certificada), IDT]", Ciudad de La Habana: Oficina Nacional de Normalización (NC), 2015.

[8] Oficina Territorial de Normalización de Villa Clara. "Adiestramiento: Las normas de la familia NC ISO 9000. Aplicación práctica". Villa Clara, 2016.

[9] I. Moreno-Montes de Oca, M. Snoeck, H.A. Reijers y A. Rodríguez-Morffi. "A systematic literature review of studies on business process modeling quality", Information and Software Technology. Vol. 58, pp. 187-205. 2015.

[10] R. Cespón Castro. "Administración de la cadena de suministros. Manual para estudiantes, académicos y empresarios vinculados al campo de la Logística.", Villa Clara. Universidad Central "Marta Abreu" de las Villas, 2011.

[11] F.D. Davis. "Perceived Usefulness, Perceived Ease of Use, and User Acceptance of Information Technology", MIS Quarterly. Vol. $13 \mathrm{~N}^{\circ}$ 3, pp. 319-340. 1989.

[12] F.D. Davis. "User acceptance of information technology: system characteristics, user perceptions and behavioral impacts". Int. J. Man - Machine Studies, pp. 475-487. 1993.

[13] G. Salles, M. Fantinato, M. Nishijima y J.P. de Albuquerque. "A Contribution to Organizational and Operational Strategic Alignment: Incorporating Business Level Agreements into Business Process Modeling", IEEE 10th International Conference on Services Computing, 2013.

[14] P.H. de Souza Bermejo, A. Luiz Zambalde, A. Olímpio Tonelli, S. Alyne Souza, L. Avelino Zuppo y P. Luiz Rosa, "Agile principles and achievement of success in software development: A quantitative study in Brazilian organizations". Procedia Technology. Vol. 16, pp. 718-727. 2014.

[15] V. Montequin, S. Cousillas, F. Ortega y J. Villanueva. "Analysis of the success factors and failure causes in Information \& Communication Technology (ICT) projects in Spain". Procedia Technology. Vol. 16, pp. 992-999. 2014.

[16] C. Jones, J. Motta y M.V. Alderete. "Gestión estratégica de tecnologías de información y comunicación y adopción del comercio electrónico en Mipymes de Córdoba, Argentina". Estudios gerenciales. Vol. 32, pp. 4-13. 2016.

[17] M.Y. Leyva Vázquez, K. Pérez Teruel, A. Febles Estrada y J. Gulín González. "Mapas cognitivos difusos para la selección de proyectos de tecnologías de la información". Contaduría y Administración. Vol. $58 \mathrm{~N}^{\circ} 4$, pp. 95-117. 2013.

[18] B. Boehm. "Value-Based Software Engineering". Software Engineering Notes. Vol. $28 \mathrm{~N}^{\circ}$ 2. 2003.

[19] E. Brynjolfsson and L.M. Hitt. "Beyond Computation: Information Technology, Organizational Transformation and Business Performance". Journal of Economic Perspective. Vol. $14 \mathrm{~N}^{\circ} 4$, pp. 23-48. 2000.

[20] A.G. Silvius. "Business \& IT Alignment in theory and practice". Proceedings of the 40th Annual Hawaii International Conference on System Sciences. IEEE Computer Society. 2007.

[21] A. Cuesta. "Tecnología de Gestión de Recursos Humanos". Academia, La Habana. 2005.

[22] R.A. Espín Andrade, R. Bello Pérez, A. Cobo Ortega, J. Marx Gómez y A. Racet Valdés. "Soft Computing for Business Intelligence". Berlin: Springer. 2014.

[23] R. Espin Andrade, G. Mazcorro Téllez, E. Fernández González, J. Marx-Gómez y M. Ines Lecich, "Compensatory Logic: A fuzzy normative model for decision making". Revista Investigación Operacional. Vol. 27 $\mathrm{N}^{\circ}$ 2, pp. 178-193. 2006.

[24] R. Stoica y P. Brouse. "IT project failure: A proposed four-phased adaptive multi-method approach". Computer Science. Vol. 16, pp. 728-736. 2013. 
[25] L. Pérez Quintero, J. Carrera Ortega, A. M. García Pérez y M.E. Maciá Gravier. "Herramienta CAPYROX: su aplicación en la definición de un proceso de gestión del conocimiento". Congreso Internacional de Información. INFO 2016. 2016.
[26] J. Carrera Ortega y L. Pérez Quintero. "CAPYROX: Una herramienta útil para el modelado de procesos orientado al valor", Informática 2016. IV Taller Internacional: Las TIC en la Gestión de las Organizaciones, 2016. 